\title{
Cell Death and Proliferation after Treatment and Reinfection of Clonorchis sinensis in the Sprague-Dawley Rat Bile Duct
}

\author{
Byoung-Hoon Min, Ka-Young Ahn ${ }^{1}$, Haeng-Sook Lee ${ }^{1}$, Soo-Jin Kim ${ }^{2, *}$, Kyoung-Hwan Joo ${ }^{1, *}$ \\ Xenotransplantation Research Center, Seoul National University College of Medicine, Seoul 110-799, Korea \\ ${ }^{1}$ Department of Parasitology and the Institute of Travel Medicine, Korea University College of Medicine, Seoul 136-705, Korea \\ ${ }^{2}$ Department of Life Science, College of Natural Science, Hallym University, Chuncheon 200-702, Korea
}

*Correspondence to:

Kim SJ,

Tel: +82-33-248-2091

Fax: +82-33-256-3420

E-mail: sjkim@hallym.ac.kr

Joo $\mathrm{KH}$,

Tel: +82-2-920-6161,

Fax: +82-2-924-4905

E-mail: kyhwjoo@korea.ac.kr

Received April14, 2015

Revised May 13, 2015

Accepted May 13, 2015
The structural change and distribution of mitochondrial enzyme (ATPase, cytochromec-oxidase), cell proliferation (proliferating cell nuclear antigen, PCNA), cell death (caspase-3) and cell growth factor (fibroblast growth factor 8, FGF-8) in the SpragueDawley rat bile duct during Clonorchis sinensis infection was investigated. Experimental groups were divided into $C$. sinensis infection, superinfection and reinfection of $C$. sinensis after 'praziquantel' treatment group. As a result, C. sinensis infected rat bile ducts showed the features of chronic clonorchiasis, i.e., connective tissue thickening, ductal fibrosis and epithelial tissue dilatation. PCNA for cell proliferation increased in the infection group, and decreased after praziquantel treatment. Caspase- 3 was distributed in reinfection group only. FGF- 8 was distributed in the rat bile duct after praziquantel treatment but not distributed in infection and reinfection group. Overall, C. sinensis infection causes physical and chemical irritations and then brings on the abnormalities of intracellular energy metabolism and cellular growth factors, which hinders bile duct tissue from functioning properly, and resultingly, fibrosis occurs and epithelial cells dilated abnormally. More intense infection makes tissue fibrosis chronical and activates apoptosis factors.

Key Words: Clonorchis sinensis, Clonorchiasis, Re-epithelialization

\section{INTRODUCTION}

Clonorchis sinensis is a kind of liver flukes that are parasitic in the bile duct. The infection with $C$. sinensis leads to physical and chemical irritation, which brings on cholangitis and also the proliferation of bile duct epithelial cells around the parasite, desquamation, and inflammation and fibrosis around the bile duct (Hou, 1955; Bhamarapravati et al., 1978; Lee et al., 1978; Min \& Soh, 1986; Cha et al., 1991). Liver fluke infection leads to the irregular dilatation of the bile duct, severe fibrosis around the bile duct, the papilliform proliferation of bile duct epithelial cells, and the proliferation in the mucous gland of the bile duct wall (Kim et al., 1999; Watanapa \& Watanapa, 2002). A study reported that once infected with $C$. sinensis, bile ducts might not to return to their original state, even though they were treated for the infection (Choi et al., 2004).

The reason why it is hard to restore it to the original state can be explained by a report that bile duct fibroblast divide more slowly than normal fibroblast when the bile duct has sustained physicochemical damage and they are different in the pattern of expression of cytoplasmic processes (Kim \& Min, 2004). Another explanation is that fibroblastic variant caused by infection with $C$. sinensis affects cytochrome-c-oxidase and porin and thus causes mitochondrial hypofucntion (Kim et al., 2007). In addition, it decreases the mitochondrial electron transferase in the bile duct epithelial tissue that multiplies abnormally (Min et al., 2010).

So far, many studies have dealt with the diseases of bile ducts infected with $C$. sinensis, but there have been few studies on reinfection with $C$. sinensis and the consequent proliferation or apoptosis in bile duct tissue and the pattern of morphological

(a) This is an open-access article distributed under the terms of the Creative Commons Attribution Non-Commercial License (http://creativecommons.org/licenses/by-nc/4.0) which permits unrestricted noncommercial use, distribution, and reproduction in any medium, provided the original work is properly cited.

Copyrights @ 2015 by Korean Society of Microscopy 
changes. This study researched into changes that would occur in bile ducts that were reinfected with $C$. sinensis after being treated for the first infection, i.e., morphological changes, and changes in the distribution of mitochondrial electron transferase such as cytochrome-c-oxidase subunit II (COXII) and adenosine triphosphate synthase (ATPase), proliferating cell nuclear antigens (PCNAs), caspase-3 (a apoptosis factor), fibroblast growth factor 8 (FGF-8), etc.

\section{MATERIALS AND METHODS}

\section{Preparation of C. sinensis Metacercaria and Experimental Animal}

Pseudorasbora parva were digested by artificial gastric juice at $37^{\circ} \mathrm{C}$ for 6 hours, and then they were filtered and rinsed several times, from which $C$. sinensis metacercariae were separated. The separated 50 metacercariae were orally administered into each Sprague-Dawley (SD) rats (female, 8 weeks). The SD rats were raised for 14 to 24 weeks to the experiment schedule. Bile ducts were extracted 4 weeks, 14 weeks, 18 weeks, and 24 weeks after parasite infection, and were examined for infection with $C$. sinensis. The experiment was conducted only on the bile ducts infected with $C$. sinensis
(Korea University, KUIACUC-20101001-1).

\section{Experimental Group}

Experimental groups were as follows, and each group was composed of $5 \mathrm{SD}$ rats (Fig. 1).

\section{Immunohistochemistry}

The extracted bile duct was fixed in 10\% buffered neutral formalin ( $\mathrm{pH}$ 7.4) and was ethanol-dehydrated, and then was paraffin-embedded. The paraffin-embedded tissue was cut into thin sections, on which deparaffinization, rehydration, antigen retrieval and peroxidase and protein blocking were conducted. The slide was reacted to primary antibodies to COXII (MS405; Mitoscience, USA) and ATPase (MS505; Mitoscience). EnVision ${ }^{\mathrm{TM}}+$ Kits (K5007; Dako, USA) was used for secondary antibodies and DAB (3,3'-diaminobenzidine) colorization. Zytochem Plus Double Stain Kit (POLDS-006; Zytomed, USA), DAB Substrate Kit (DAB057; Zytomed), and Fast-Red Substrate Kit (RED055; Zytomed) were used for the double staining of PCNA (CBL407; Chemicon, USA) and caspase-3 (9664S; Cell Signaling Technology, USA), PCNA and FGF-8. The prepared slide was observed through a light microscope (Zeiss Axio Scope; Zeiss, Germany) and the image

\begin{tabular}{|c|c|c|c|c|c|}
\hline Group A & $\begin{array}{l}\text { Non-infection } \\
\downarrow \\
\end{array}$ & & & & \\
\hline Group B & $\begin{array}{l}\text { Non-infection } \\
\downarrow \\
\end{array}$ & $\begin{array}{l}\text { MC50 infection } \\
\downarrow\end{array}$ & $\begin{array}{l}\text { Worm recovery } \\
\downarrow\end{array}$ & & \\
\hline Group C & $\begin{array}{l}\text { MC50 infection } \\
\downarrow\end{array}$ & $\begin{array}{l}\text { MC50 superinfection } \\
\downarrow\end{array}$ & $\begin{array}{l}\text { Worm recovery } \\
\downarrow\end{array}$ & & \\
\hline Group D & $\begin{array}{l}\text { MC50 infection } \\
\downarrow\end{array}$ & $\begin{array}{l}\text { Tx, MC50 reinfection } \\
\downarrow\end{array}$ & $\begin{array}{l}\text { Worm recovery } \\
\downarrow\end{array}$ & & \\
\hline Group E & $\begin{array}{l}\text { MC50 infection } \\
\downarrow\end{array}$ & $\begin{array}{l}\text { Tx } \\
\downarrow \\
\end{array}$ & $\begin{array}{l}\text { MC50 reinfection } \\
\downarrow\end{array}$ & $\begin{array}{l}\text { Worm recovery } \\
\downarrow\end{array}$ & \\
\hline Group F & $\begin{array}{l}\text { MC50 infection } \\
\downarrow\end{array}$ & $\begin{array}{l}\text { Tx } \\
\downarrow \\
\end{array}$ & $\begin{array}{l}\text { MC50 reinfection } \\
\downarrow\end{array}$ & & $\begin{array}{l}\text { Worm recovery } \\
\downarrow\end{array}$ \\
\hline \multirow[t]{2}{*}{ Group G } & $\begin{array}{l}\text { Non-infection } \\
\downarrow \\
\end{array}$ & $\begin{array}{l}\text { Tx } \\
\downarrow \\
\end{array}$ & & \multirow[b]{2}{*}{18 weeks } & \multirow[b]{2}{*}{24 weeks } \\
\hline & 0 week & 10 weeks & 14 weeks & & \\
\hline
\end{tabular}

Fig. 1. Scheme of experimental group. Group A: Normal control group. Group B: Sprague-Dawley (SD) rat bile duct at 4 weeks after Clonorchis sinensis infection. Group C: SD rat bile duct at 14 weeks after C. sinensis superinfection. Group D: SD rat bile duct at 14 weeks after $C$. sinensis infection. C. sinensis were infected at 0 week and treated with praziquantel at 10 weeks after infection and then reinfection of $C$. sinensis was performed immediately. Group E: SD rat bile duct at 18 weeks after $C$. sinensis infection. C. sinensis were infected at 0 week and treated with praziquantel at 10 weeks after infection. Reinfection was performed at 4 weeks after praziquantel treatment. Group F: SD rat bile duct at 24 weeks after C. sinensis infection. C. sinensis were infected at 0 week and treated with praziquantel at 10 weeks after infection. Reinfection was performed at 4 weeks after praziquantel treatment. Group G: Praziquantel administration without infection. MC, metacercariae; Tx, treatment. 
was photographed through a digital camera (Zeiss AxioCam; Zeiss). Tissue analysis was conducted by MetaMorph software (Universal Imaging Co., USA).

\section{Transmission Electron Microscopy}

The extracted bile duct was fixed in $1 \%$ paraformaldehyde, $1 \%$ glutaraldehyde and 2\% osmium tetroxide. The fixed tissue was ethanol-dehydrated and was embedded with lowicryl HM20. Then, the embedded tissue was cut into ultrathin sections $(60 \mathrm{~nm})$ by the use of an ultramicrotome (Ultracut E; Reichert-Jung, USA), and the ultrathin sections were stained with uranyl acetate and lead citrate and were observed through a transmission electron microscope (Zeiss EM 109; Zeiss).

\section{RESULTS}

\section{Morphological Change of Infected Bile Ducts}

Normal bile ducts were about $1.04 \mathrm{~mm}$ thick (Fig. 2A), but bile ducts infected with $C$. sinensis in the group B were about $5.68 \mathrm{~mm}$ thick (Fig. 2B). The bile ducts of the group $\mathrm{C}$ were $6.21 \mathrm{~mm}$ thick on average. Those of the group D were reinfected with $C$. sinensis after being treated for the first infection; they were $5.72 \mathrm{~mm}$ thick on average (Fig. 2C). Those of the group $\mathrm{E}$ and $\mathrm{F}$, left for certain periods of time, were $4.79 \mathrm{~mm}$ and $4.32 \mathrm{~mm}$ thick, respectively.

For connective tissue and epithelial tissue constituting the bile duct, their thicknesses were comparatively analyzed in terms of infection aspect. The bile ducts of the group B, left for 4 weeks after being infected, became about 546\% thicker than normal. Connective tissue and epithelial tissue expanded by $264 \%$ and $1,468 \%$, respectively. Those of the group C, left for 14 weeks after being infected, were similar in thickness to those of the group B. Those of the group D, reinfected with $C$. sinensis metacercariae immediately after being treated for the first infection with anthelmintic 'praziquantel', were similar in thickness to those of group B and C that were not treated for C. sinensis infection. However, connective tissue and epithelial tissue became about 18\% thinner. In the group E, bile ducts were reinfected with $C$. sinensis 4 weeks after being treated for the first infection, and then they remained infected for 4 weeks. They became about $460 \%$ thicker than normal. The bile ducts of the group F, which remained infected for 10 weeks, became about $415 \%$ thicker than normal. Those of the group $\mathrm{G}$ (a control group) were similar in size to normal (Table $1)$.

\section{Distribution of ATPase and COXII}

In normal bile ducts, ATPase (Fig. 3A1) and COXII (Fig. 3A2) are distributed in connective tissues, but did not distribute in epithelial tissues. In bile ducts infected with $C$. sinensis, epithelial cells increased, and in dilated connective tissue, fibrosis was observed (group B to F). The group A (a normal control group) showed that ATPase (Fig. 3A1) and COXII (Fig. 3A2) were distributed in the connective tissues of bile ducts. In the group B, ATPase and COXII (Fig. 3B1 and B2) were decreased compared to the group A. ATPase and COXII were found distributed in some cells in the base of epithelial tissue (Fig. 3B1 and B2). The groups B, C, and D showed that ATPase (Fig. 3B1, C1, and D1) and COXII (Fig. 3B2, C2, and D2) were distributed in the connective tissues of some bile ducts; especially in the group C and D, COXII was distributed mostly around the outer part of connective tissue (Fig. 3C2 and D2). The group E (Fig. 3E1 and E2) and the group F (Fig. 3F1 and F2) showed that ATPase and COXII were distributed evenly in connective tissue. The group G showed similar
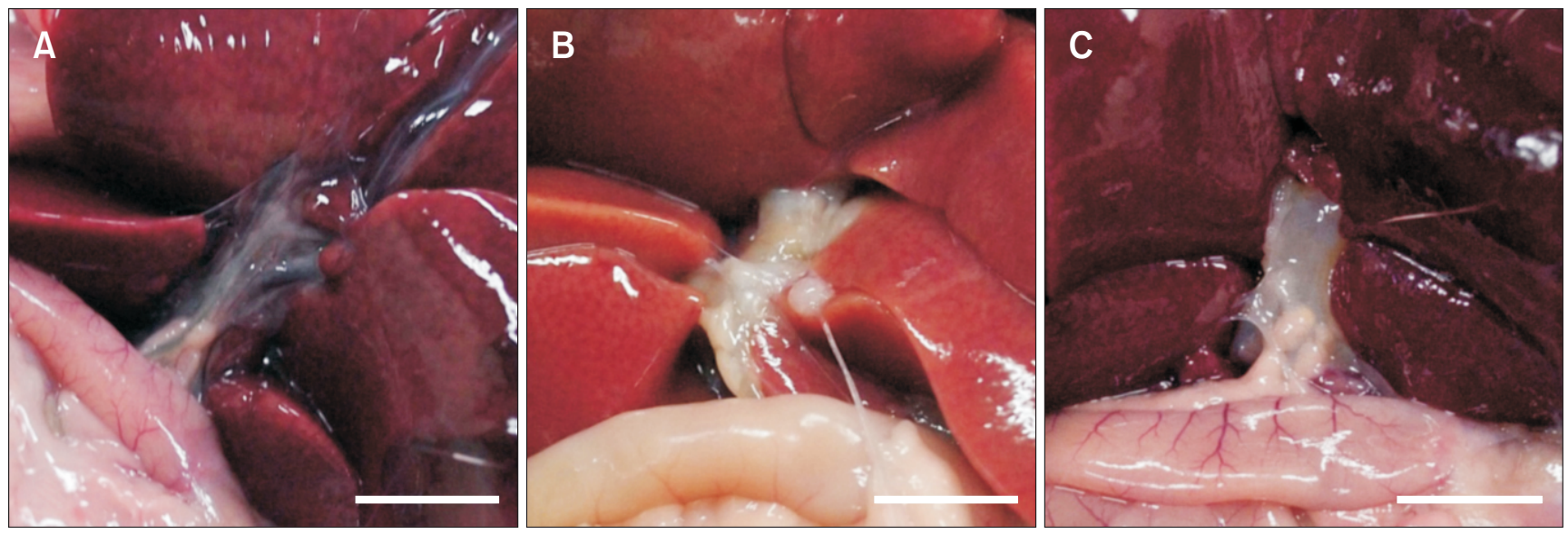

Fig. 2. Extracted abdominal organs of a Sprague-Dawley (SD) rat, showing marked dilatation of the common and extra hepatic bile duct (B, C). Bile duct obtained from praziquantel treatment group was transparent than Clonorchis sinensis infected bile duct (C). A: Normal control group (group A). B: SD rat bile duct at 4 weeks after $C$. sinensis infection (group B). C: SD rat bile duct at 18 weeks after $C$. sinensis infection. $C$. sinensis were infected at 0 week and treated with praziquantel at 10 weeks after infection. Superinfection was performed at 4 weeks after praziquantel treatment (group E). Scale bars=1 cm. 


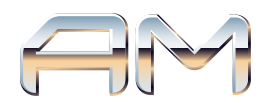

Table 1. Dilatation of Sprague-Dawley rat bile duct during Clonorchis sinensis infection and praziquantel treatment $(\mathrm{n}=5)$

\begin{tabular}{|c|c|c|c|c|c|c|c|}
\hline & Group A & Group B & Group C & Group D & Group E & Group F & Group G \\
\hline Width of bile duct (mm) & $1.04 \pm 0.1$ & $5.68 \pm 0.2$ & $6.21 \pm 0.1$ & $5.72 \pm 0.2$ & $4.79 \pm 0.4$ & $4.32 \pm 0.2$ & $1.09 \pm 0.1$ \\
\hline Thickness of connective tissue $(\mu \mathrm{m})$ & $50.5 \pm 4.3$ & $133.9 \pm 29.2$ & $137.1 \pm 24.2$ & $118.1 \pm 44.7$ & $147.9 \pm 24.2$ & $130.1 \pm 32.3$ & $49.4 \pm 2.1$ \\
\hline Thickness of epithelial tissue $(\mu \mathrm{m})$ & $10.2 \pm 0.3$ & $149.8 \pm 14.7$ & $155.1 \pm 15.2$ & $123.7 \pm 12.1$ & $111.1 \pm 10.9$ & $111.5 \pm 10.9$ & $11.1 \pm 1.2$ \\
\hline
\end{tabular}

Values are presented as mean \pm standard deviation.

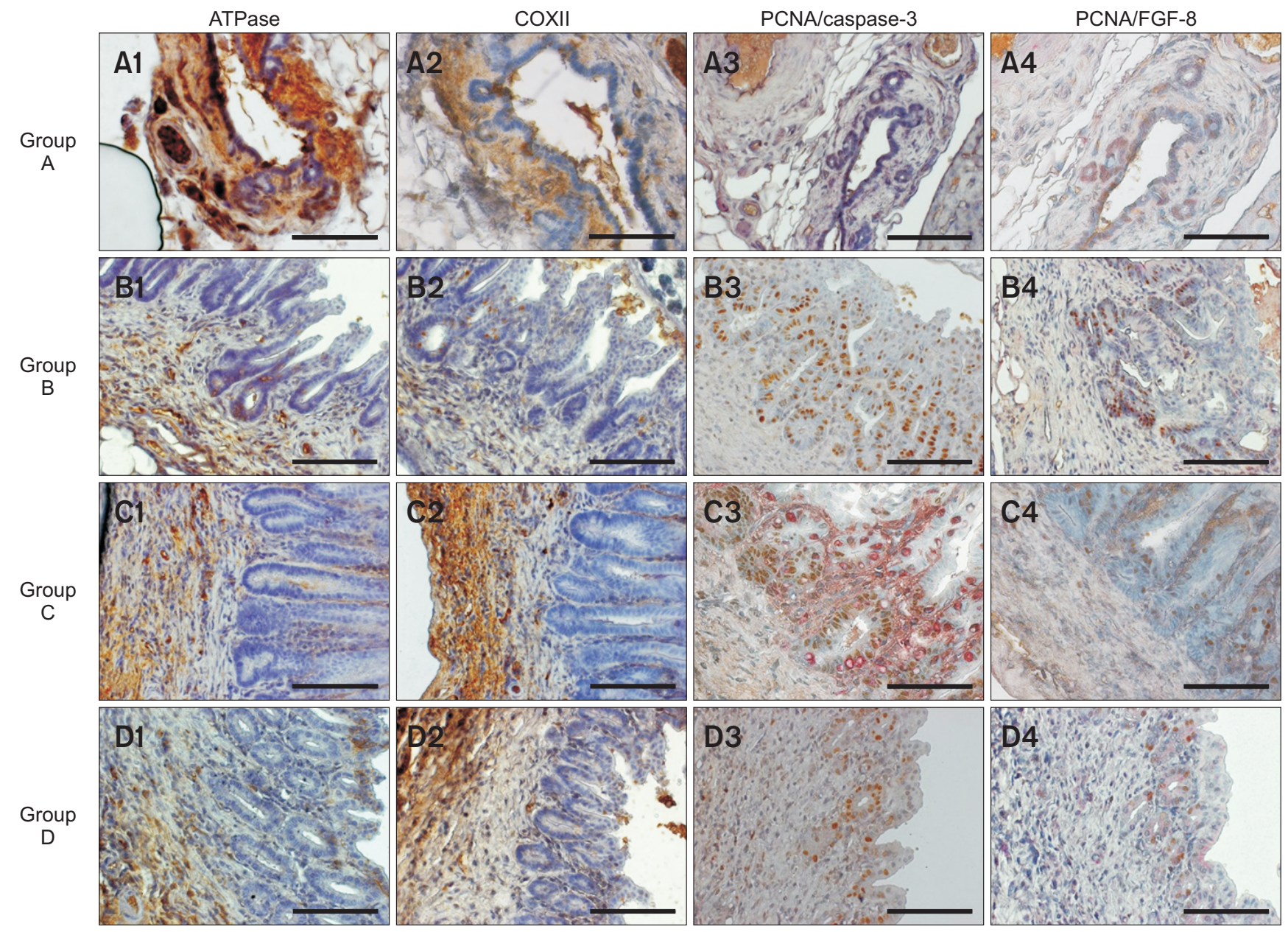

Fig. 3. Light micrograph of the Sprague-Dawley (SD) rat bile duct which was reacted with adenosine triphosphase (ATPase), cytochrome-c-oxidase subunit II (COXII), proliferating cell nuclear antigen (PCNA), caspase-3, and fibroblast growth factor 8 (FGF-8). ATPase and COXII; group B (Fig. 3B1 and B2) was decreased compared to the group A (Fig. 3A1 and A2). Groups C and D, COXII was widely distributed in connective tissue (Fig. 3C2 and D2). The group E (Fig. $3 \mathrm{E} 1$ and E2) and the group F (Fig. 3F1 and F2) showed that ATPase and COXII were distributed evenly in connective tissue. PCNA, caspase-3, and FGF8; PCNA is distributed in most epithelial tissues of group B (Fig. 3B3). Especially caspase-3 was observed only in the group C that was superinfected (Fig. 3C3). FGF-8 was found in part of epithelial tissue and connective tissue (Fig. 3D4 and E4), and was distributed widely throughout bile duct epithelial cells in the group F (Fig. 3F4). The group G showed similar results to the normal control group (Fig. 3G1-G4). Scale bars=100 $\mu \mathrm{m}$.

results to the normal control group (Fig. 3G1 and G2).

\section{Distribution of PCNA and Caspase-3}

The group A showed that PCNA was distributed partially in connective tissue (Fig. 3A3), and the group B (Fig. 3B3) showed the distribution of PCNA in most epithelial tissues excluding ones near the lumens. The group C (Fig. 3C3) showed its distribution in epithelial tissue, and caspase-3 was observed in 8.75 cells per $100 \mu \mathrm{m}^{2}$ (Table 2). The group D and E (Fig. 3D3 and E3) showed its distribution in epithelial cells near connective tissue, and its quantity was less than the group B. The group F showed its distribution in part of contracted epithelial tissue (Fig. 3F3). The group G showed similar result to the normal control group (Fig. 3G3). It was 

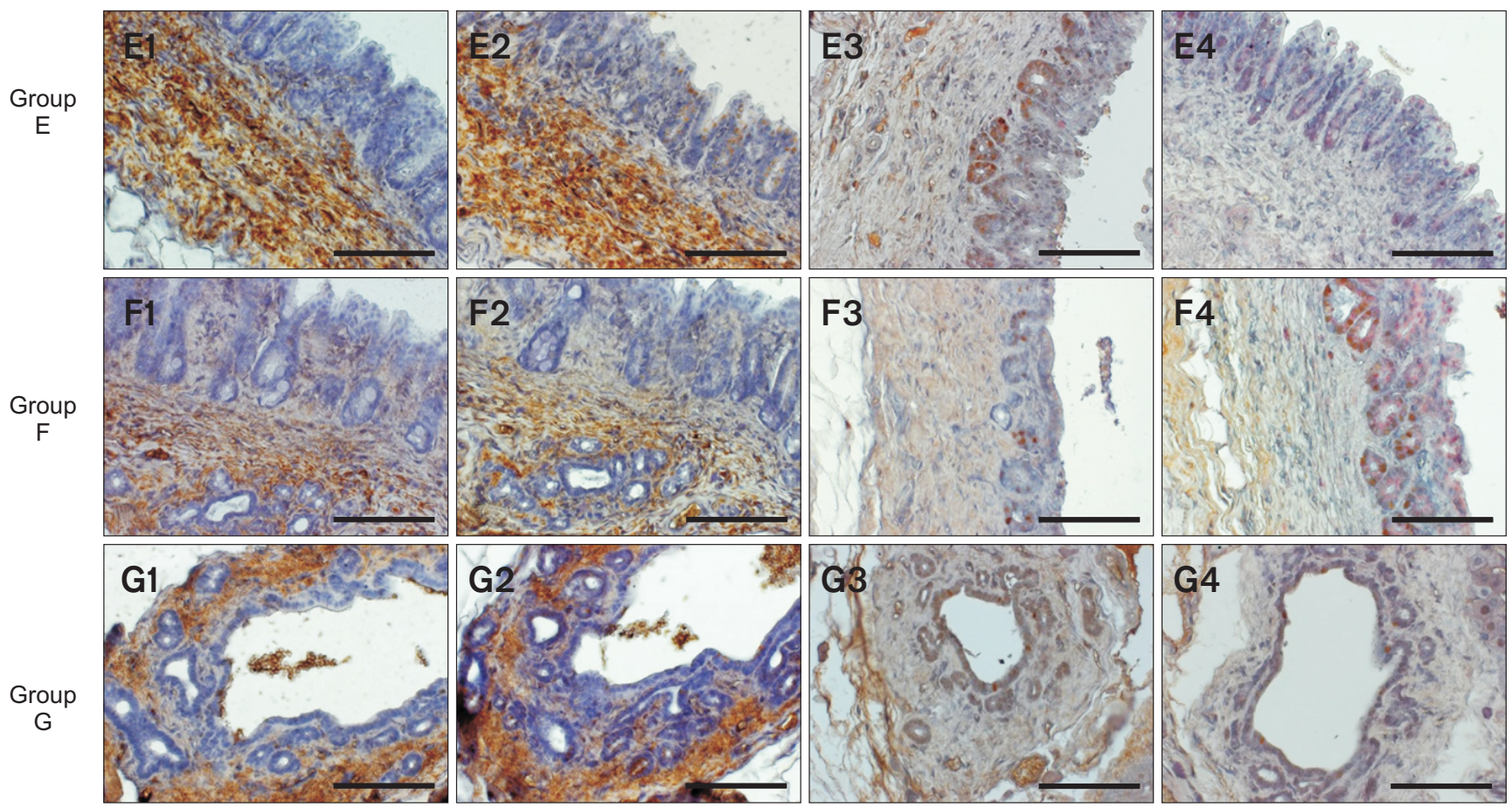

Fig. 3. Continued.

Table 2. Distribution of PCNA, caspase- 3 and FGF- $8(n=5)$

\begin{tabular}{|c|c|c|c|c|c|c|c|}
\hline \multirow{2}{*}{ Antibody } & \multicolumn{7}{|c|}{ Group } \\
\hline & A & B & $\mathrm{C}$ & $\mathrm{D}$ & E & F & G \\
\hline \multirow[t]{2}{*}{ PCNA } & + & ++++ & ++++ & +++ & +++ & ++ & + \\
\hline & $3.5 \pm 0.7$ & $24.5 \pm 16.1$ & $20.4 \pm 13.7$ & $11.7 \pm 2.8$ & $10.1 \pm 3.4$ & $5.7 \pm 1.5$ & $3.9 \pm 0.4$ \\
\hline \multirow[t]{2}{*}{ Caspase-3 } & - & - & +++ & - & - & - & - \\
\hline & & & $8.75 \pm 3.5$ & & & & \\
\hline \multirow[t]{2}{*}{ FGF-8 } & + & - & - & +++ & ++++ & ++++ & + \\
\hline & $2.3 \pm 0.5$ & & & $15.9 \pm 5.1$ & $27.3 \pm 4.7$ & $29.1 \pm 2.1$ & $1.3 \pm 0.5$ \\
\hline
\end{tabular}

Values are presented as mean \pm standard deviation.

PCNA, proliferating cell nuclear antigen; FGF-8, fibroblast growth factor 8.

The number of the each antibodies were counted from ten separated areas of the sections.

found that the group C contained PCNA most, but its PCNA decreased as time passed after the treatment for $C$. sinensis infection. The distribution of caspase- 3 was observed only in the group C that was superinfected (Table 2).

\section{Distribution of PCNA and FGF-8}

The group A showed the distribution of FGF- 8 in part of epithelial tissue (Fig. 3A4). The group B showed a large number of PCNA in the base of epithelial tissue, and FGF8 was found distributed in very few of cells (Fig. 3B4). In the group $\mathrm{C}$, the distribution of PCNA was similar to that in the group B. However, the distribution of FGF-8 was not detected (Fig. 3C4). As regards the groups D and E, PCNA in epithelial tissue decreased compared to the groups B and C. FGF-8 was found in part of epithelial tissue and connective tissue (Fig. 3D4 and E4). The group F showed that FGF-8 was distributed widely throughout bile duct epithelial cells (Fig. 3F4). It was found that the groups E and F contained FGF-8 most (Table 2).

\section{TEM Observation}

In the group A, monolayered epithelial tissue was observed (Fig. 4A). The group B showed the fibrosis of connective tissue and increase in number of epithelial cells outside connective tissue (Fig. 4B). The group C showed that epithelial cells had been damaged by fibrous tissue and by the connective tissue of bile ducts that developed fibrosis (Fig. 4C). The 

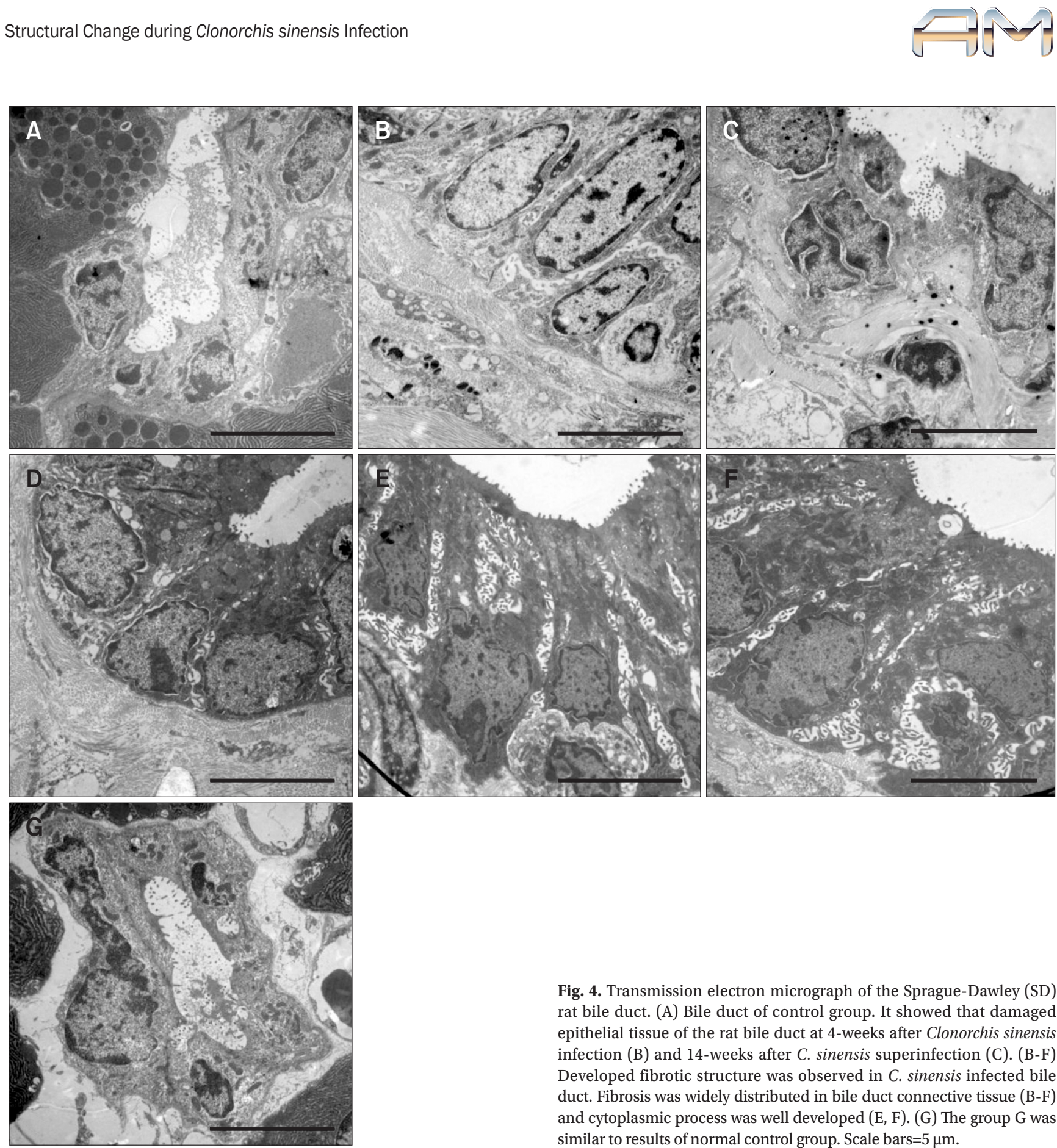

Fig. 4. Transmission electron micrograph of the Sprague-Dawley (SD) rat bile duct. (A) Bile duct of control group. It showed that damaged epithelial tissue of the rat bile duct at 4-weeks after Clonorchis sinensis infection (B) and 14-weeks after C. sinensis superinfection (C). (B-F) Developed fibrotic structure was observed in C. sinensis infected bile duct. Fibrosis was widely distributed in bile duct connective tissue (B-F) and cytoplasmic process was well developed (E, F). (G) The group G was similar to results of normal control group. Scale bars $=5 \mu \mathrm{m}$.

groups D and E showed epithelial tissue and the connective tissue of bile ducts that developed fibrosis (Fig. 4D and E). As compared to the group $\mathrm{D}$, the group $\mathrm{E}$ showed a larger number of the cytoplasmic processes of bile duct epithelial cells (Fig. 4E). The group F showed bile duct epithelial tissue with cytoplasmic processes, as in the group E (Fig. 4E). The group $\mathrm{G}$ did not show morphological difference between its bile ducts and normal ones (Fig. 4G).

\section{DISCUSSION}

This study researched into changes that would occur in bile ducts that were re-infected with $C$. sinensis after being treated for the first infection, i.e., morphological changes, and changes in the distribution of mitochondrial electron transferase such as COXII and ATPase, PCNAs, an apoptosis factor (caspase-3), FGF-8, etc.

C. sinensis infection is very common in Southeast Asian countries and Korea (Korea Association of Health Promotion, 
2009), and it causes clonorchiasis. In the process of lesion development, it causes physical and chemical irritation, leads to inflammatory cell infiltration in the mucosa and submucosa layer of the bile duct and to the proliferation and desquamation of the mucous epithelium (Rim, 1990). When infection lasts long, glandular cells and bile canaliculi newly form in the bile duct, and moreover fibrosis progresses around epithelial tissue, which dilates the bile duct and causes cystic changes (Rim, 1990; Cha et al., 1991). Also, a study reported that it was hard to restore the dilated bile duct to its original state (Choi et al., 2004).

Likewise, bile ducts used in this study were dilated by $C$. sinensis infection and could not return to their original state. On a histological examination, abnormal dilatations were observed in the connective and epithelial tissues of infected bile ducts, and also infected and re-infected bile ducts were having the thickest connective and epithelial tissues. The connective tissues of bile ducts treated for infection did not show a significant difference with those of untreated ones, but their epithelial tissues became thinner as time passed after treatment. That result may be explained by physical and chemical irritations caused by $C$. sinensis infection; such irritations seem to make connective and epithelial tissues dilated abnormally. As for the connective tissues of bile ducts infected with $C$. sinensis, their thickness hardly changes from the 4th week from infection, which lasts even after treatment. It implies that once infected, bile ducts may not return to the normal state though they are treated for infection.

Mitochondria produce ATP, necessary energy for cell metabolism, through the electron transport system. In case a problem arises in that process, a reduction occurs in ATP production efficiency (Navarro \& Boveris, 2004a; Navarro et al., 2005). Bustamante et al. (2004) reported that apoptosis could occur when mitochondria worked abnormally. Moreover, it was reported that mitochondrial electron transferase decreased in bile duct connective tissue that had dilated abnormally after C. sinensis infection (Kim et al., 2007; Min et al., 2010).

Mitochondrial electron transferases such as ATPase and COXII were being evenly distributed in the connective tissues of normal bile ducts, and so they were having normal metabolisms. In contrast, in bile ducts infected with $C$. sinensis, fibrous tissue increases and electron transferases decreased. In the connective tissues of bile ducts treated for infection, ATPase and COXII increased in distribution. It may be related to a previous report that in bile duct tissue infected with $C$. sinensis, fibroblastic variant caused by the morphological damage of mitochondria and a reduction in the distribution of electron transferase, maintained the fibrosis of bile duct connective tissue (Min et al., 2010). In other words, it is presumed that fibrosis lasts due to the functional anomaly of fibroblasts in damaged connective tissue, even though C. sinensis infection has been cured. The PCNA, used to observe the proliferation and apoptosis of cells, is a cofactor for DNA polymerases and is known as an important factor for DNA replication and repair. In immunohistochemical experiments, it is used for the marker of cell growth factors (Bacchi \& Gown, 1993; Onuma et al., 2001; Paunesku et al., 2001). Caspase-3 is an antigen that is expressed before morphological changes such as karyoclasis, a process of apoptosis, occur and is used to confirm apoptosis (Krajewska et al., 1997; Duan et al., 2003; Bulut et al., 2006).

In this study, cells remarkably proliferated in the epithelial cells of bile ducts infected with $C$. sinensis, bile duct epithelial cells decreased in number, and PCNA expression was reduced as time passed after treatment. However, such changes were not observed in normal bile ducts, ones left for 4 weeks after infection, and ones treated for infection. In the case of caspase-3, the widest distribution was observed in bile ducts that were infected again 10 weeks after the first infection. That result may be explained as follows: as $C$. sinensis directly irritated the bile duct, epithelial cells aggressively proliferated, which resulted in the dilatation of bile duct epithelial tissue. Also, that result seems to be related to animal studies that reported the proliferation of bile duct epithelial cells that were all induced by the ischemia or ligation of the bile duct (Alpini et al., 1989, Beaussier et al., 2005), the fibrosis of bile ducts infected with C. sinensis, the dilatation of connective tissue, and the increase in epithelial cells (Min et al., 2010). But in bile ducts of which $C$. sinensis infection was cured, bile duct epithelial tissue seems to hardly proliferate even though $C$. sinensis irritation vanished. In bile ducts reinfected 4 weeks after the first infection, infection becomes more intense, which means that $C$. sinensis gives physical and chemical irritations more strongly. As a result, it seems that epithelial tissue may be destroyed by stronger irritations. But during early reinfection, apoptosis is reduced. It may be because bile duct epithelial tissue is dilated during early reinfection and comparatively milder irritation is given to bile ducts treated for the first infection.

A wound is healed up after three stages, inflammation, granulation tissue formation and re-epithelialization (Singer \& Clark, 1999; Clark, 2001). In particular, the FGF is known to get involved in tissue growth and repair, e.g., embryo growth, skeletal growth, wound repair, vasculogenesis and inflammation control (Xiao et al., 2007; Turner \& Grose, 2010). During this study, FGF-8 was not observed in the epithelial tissues of bile ducts infected with $C$. sinensis. Contrarily, in bile ducts treated for infection, FGF- 8 was observed and tended to increase with time. Thus, in infected bile ducts, the parasites give more intense irritation and thus epithelial tissue proliferates more aggressively. It seems that after they were treated for $C$. sinensis infection with 'praziquantel', bile duct epithelial tissue begins to repair the 
wounds. Electron microscopy also showed that the epithelial cells of infected bile ducts had been damaged by fibrous tissue, and that connective tissue had developed fibrosis. In bile ducts treated for infection, epithelial cells were not damaged and cytoplasmic processes were well developed. These results imply that the epithelial cells of bile ducts given treatment get growth and repair, unlike those of infected bile ducts that repeatedly proliferate after infection.

It was reported that apoptosis could occur in cases where mitochondria inhibited ATP synthesis (Cottrell \& Turnbull, 2000; Navarro \& Boveris, 2004a; Navarro et al., 2002, 2004b, 2005; Dirks et al., 2006), where intracellular nitric oxide increased (mitochondria-dependent apoptosis) (Bustamante et al., 2004), and where mitoptosis (mitochondria suicide) occurred due to the malfunction of mitochondria (Skulachev, 2006).

This study found that ATPase and cytochrome-c-oxidase decreased in the connective tissues of bile ducts infected with C. sinensis, PCNA increased in their epithelial tissues, and caspase- 3 increased in the epithelial tissues of re-infected bile ducts. These results are presumed to be caused by the fibrosis of bile duct connective tissue and the abnormal dilatation of epithelial tissue. In particular, increases in cytochrome-coxidase and caspase- 3 seem to be related to following reports: mitochondria-dependent apoptosis causes abnormalities in the electron transfer system, by which cytochrome-c-oxidase is emitted and caspase-3 is activated (Duke et al., 1996; Green \& Kroemer, 1998; Senaratne \& Colston, 2002; Bustamante et al., 2004); apoptosis occurs when ATP is not produced by the deficiency of mitochondrial enzymes (Dirks et al., 2006; Skulachev, 2006).

C. sinensis infection causes physical and chemical irritations and then brings on the abnormalities of intracellular energy metabolism and cellular growth factors, which hinders bile duct tissue from functioning properly, and resultingly, fibrosis occurs and epithelial cells dilated abnormally. More intense infection makes tissue fibrosis chronical and activates apoptosis factors such as caspase- 3 that destroy bile duct epithelial cells. But in case infection is cured and physical and chemical irritations wear off, it decreases abnormal growth factors that act on bile duct epithelial tissue and activates cell growth factors such as FGF-8 that are necessary for normal metabolism. As a result, it enables cells that form the connective and epithelial tissues of a bile duct to have normal metabolisms.

\section{SUMMARY}

This study researched into changes that would occur in bile ducts that were reinfected with $C$. sinensis after being treated for the first infection, i.e., morphological changes, and changes in the distribution of mitochondrial electron transferase (ATPase, cytochrome-c-oxidase), PCNAs, apoptosis factor (caspase-3), FGF-8, etc. As a result, C. sinensis infected rat bile ducts showed the features of chronic clonorchiasis including connective tissue thickening, ductal fibrosis and epithelial tissue dilatation. PCNA was increased in the infection group, and decreased after praziquantel treatment. Caspase- 3 was distributed in reinfection group only. FGF-8 was distributed in the rat bile duct after praziquantel treatment but not distributed in infection and reinfection group.

Overall, $C$. sinensis infection causes physical and chemical irritations and then brings on the abnormalities of intracellular energy metabolism and cellular growth factors, which hinders bile duct tissue from functioning properly, and resultingly, fibrosis occurs and epithelial cells dilated abnormally. More intense infection makes tissue fibrosis chronical and activates apoptosis factors.

\section{CONFLICT OF INTEREST}

No potential conflict of interest relevant to this article was reported.

\section{REFERENCES}

Alpini G, Lenzi R, Zhai W R, Slott P A, Liu M H, Sarkozi L, and Tavoloni N (1989) Bile secretory function of intrahepatic biliary epithelium in the rat. Am. J. Physiol. 257, 124-133.

Bacchi C E and Gown A M (1993) Detection of cell proliferation in tissue sections. Braz. J. Med. Biol. Res. 26, 677-687.

Beaussier M, Wendum D, Fouassier L, Rey C, Barbu V, Lasnier E, Lienhart A, Scoazec J Y, Rosmorduc O, and Housset C (2005) Adaptative bile duct proliferative response in experimental bile duct ischemia. $J$. Hepatol. 42, 257-265.

Bhamarapravati N, Thamavit W, and Vajrasthira S (1978) Liver changes in hamsters infected with a liver fluke of man, Opisthorchis viverini.
Am. J. Trop. Med. Hyg. 27, 787-794.

Bulut S, Uslu H, Ozdemir B, and Bulut O (2006) Expression of caspase-3, p53 and $\mathrm{Bcl}-2$ in generalized aggressive periodontitis. Head Face Med. 2, 17.

Bustamante J, Di L E, Fernandez-Cobo M, Monti N, Cadenas E, and Boveris A (2004) Kinetic analysis of thapsigargin-induced thymocyte apoptosis. Free Radic. Biol. Med. 37, 1490-1498.

Cha S H, Lee J H, and Rim H J (1991) Histopathological changes of the bile duct in the experimental anivals by the superinfection of Clonorchis sinensis. Korea Univ. Med. J. 28, 741-757. 
Choi D I, Hong S T, Li S, Chung B S, Lim J H, and Lee S H (2004) Bile duct changes in rats reinfected with Clonorchis sinensis. Korean J. Parasitol. 42, 7-17.

Clark R A (2001) Fibrin and wound healing. Ann. NY. Acad. Sci. 936, 355-367.

Cottrell D A and Turnbull D M (2000) Mitochondria and ageing. Curr. Opin. Clin. Nutr. Metab. Care 3, 473-478.

Dirks A J, Hofer T, Marzetti E, Pahor M, and Leeuwenburgh C (2006) Mitochondrial DNA mutations, energy metabolism and apoptosis in aging muscle. Ageing Res. Rev. 5, 179-195.

Duan W R, Garner D S, Williams S D, Funckes-Shippy C L, Spath I S, and Blomme E A (2003) Comparison of immunohistochemistry for activated caspase-3 and cleaved cytokeratin 18 with the TUNEL method for quantification of apoptosis in histological sections of PC-3 subcutaneous xenografts. J. Pathol. 199, 221-228.

Duke R C, Ojcius D M, and Young J D (1996) Cell suicide in health and disease. Sci. Am. 275, 80-87.

Green D and Kroemer G (1998) The central executioners of apoptosis: caspases or mitochondria? Trends. Cell Biol. 8, 267-271.

Hou P C (1995) The pathology of Clonorchis sinensis infestation of the liver. J. Pathol. Bacteriol. 70, 53-64.

Kim K H, Kim C D, Lee H S, Lee S J, Jeen Y T, Chun H J, Song C W, Lee S W, Um S H, Choi J H, Ryu H S, and Hyun J H (1999) Biliary papillary hyperplasia with clonorchiasis resembling cholangio- carcinoma. Am. J. Gastroenterol. 94, 514-517.

Kim S J, Lee B W, Oh J Y, and Joo K H (2007) The distribution of porin and cytochrome-c-oxidase on mitochondrial cristae on the cultured fibroblast of rat bile duct, (KSEM 38th Symposium, Daejeon).

Kim S J and Min B H (2004) Ultrastructural change of the bile duct fibroblast at infected rat with Clonorchis sinensis. Korean J. Electron. Microscopy 34, 121-130.

Korea Association of Health Promotion (2009) Prevalence of Intestinal Parasitic Infections in Korea, 7th Report (Korea Association of Health Promotion, Seoul).

Krajewska M, Wang H G, Krajewski S, Zapata J M, Shabaik A, Gascoyne $\mathrm{R}$, and Reed J C (1997) Immunohistochemical analysis of in vivo patterens of expression of CPP32 (caspase-3), a cell death protease. Cancer Res. 57, 1605-1613.

Lee S H, Shim T S, and Lee S M (1978) Studies on pathological changes of the liver in albino rats infected with Clonorchis sinensis. Korean J. Parasitol. 16, 148-155.

Min B H, Hong S H, Lee H S, Kim S J, and Joo K H (2010) Reduction of mitochondrial electron transferase in rat bile duct fibroblast by
Clonorchis sinensis infection. Korean J. Microscopy 40, 89-99.

Min H K and Soh C T (1986) The effect of a carcinogen, dimethylnitrosamin, in cholangiocarcinoma in the albino rats experimentally infected with Clonorchis sinensis metacercaria. Yonsei Rep. Trop. Med. 17, 1-10.

Navarro A and Boveris A (2004a) Rat brain and liver mitochondria develop oxidative stress and lose enzymatic activities on aging. Am. J. Physiol. Regul. Integr. Comp. Physiol. 287, 1244-1249.

Navarro A, Gomez C, Lopez-Cepero J M, and Boveris A (2004b) Beneficial effects of moderate exercise on mice aging: survival, behavior, oxidative stress, and mitochondrial electron transfer. Am. J. Physiol. Regul. Integr. Comp. Physiol. 286, 505-511.

Navarro A, Gomez C, Sanchez-Pino M J, Gonzalez H, Bandez M J, Boveris $A$ D, and Boveris A (2005) Vitamin E at high doses improves survival, neurological performance, and brain mitochondrial function in aging male mice. Am. J. Physiol. Regul. Integr. Comp. Physiol. 289, 13921399.

Navarro A, Sanchez-Pino M J, Gomez C, Peralta J L, and Boveris A (2002) Behavioral dysfunction, brain oxidative stress, and impaired mitochondrial electron transfer in aging mice. Am. J. Physiol. Regul. Integr. Comp. Physiol. 282, 985-992.

Onuma H, Mastui C, and Morohashi M (2001) Quantitative analysis of the proliferation of epidermal cells using a human skin organ culture system and the effect of DbcAMP using markers of proliferation (BrdU, Ki-67, PCNA). Arch. Dermatol. Res. 293, 133-138.

Paunesku T, Mittal S, Protic M, Oryhon J, Korolev S V, Joachimiak A, and Woloschak G E (2001) Proliferating cell nuclear antigen (PCNA): ringmaster of the genome. Int. J. Radiat. Biol. 77, 1007-1021.

Rim H J (1990) Clonorchiasis in Korea. Korean J. Parasitol. 28(Suppl), 63-78.

Senaratne S G and Colston K W (2002) Direct effects of bisphosphonates on breast cancer cells. Breast. Cancer Res. 4, 18-23.

Singer A J and Clark R A (1999) Cutaneous wound healing. N. Engl. J. Med. 341, 738-746.

Skulachev V P (2006) Bioenergetic aspects of apoptosis, necrosis and mitoptosis. Apoptosis 11, 473-485.

Turner N and Grose R (2010) Fibroblast growth factor signalling: from development to cancer. Nat. Rev. Cancer 10, 116-129.

Watanapa P and Watanapa W B (2002) Liver fluke-associated cholangiocarcinoma. Br. J. Surg. 89, 962-970.

Xiao Y T, Xiang L X, and Shao J Z (2007) Bone morphogenetic protein. Biochem. Biophys. Res. Commun. 362, 550-553. 